\title{
A novel intragenic deletion in OPHN1 in a Japanese patient with Dandy-Walker malformation
}

\author{
Aritoshi lida (1) ', Eri Takeshita ${ }^{2}$, Shunichi Kosugi ${ }^{3}$, Yoichiro Kamatani $\mathbb{1}^{3,4}$, Yukihide Momozawa ${ }^{5}$, Michiaki Kubo ${ }^{6}$, \\ Eiji Nakagawa², Kenji Kurosawa ${ }^{7}$, Ken Inoue ${ }^{8}$ and Yu-ichi Goto ${ }^{8,9}$
}

\begin{abstract}
Dandy-Walker malformation (DWM) is a rare congenital malformation defined by hypoplasia of the cerebellar vermis and cystic dilatation of the fourth ventricle. Oligophrenin-1 is mutated in X-linked intellectual disability with or without cerebellar hypoplasia. Here, we report a Japanese DWM patient carrying a novel intragenic 13.5-kb deletion in OPHN1 ranging from exon 11-15. This is the first report of an OPHN1 deletion in a Japanese patient with DWM.
\end{abstract}

Dandy-Walker malformation (DWM) is a midbrain-hindbrain malformation characterized by cerebellar vermis hypoplasia and dysplasia, cystic dilatation of the fourth ventricle and an elevated torcula, often accompanied by hydrocephalus ${ }^{1}$. The frequency of DWM in the U.S. is $\sim 1$ in 25,000-35,000 liveborn infants (https://rarediseases.org/rare-diseases/dandy-walkermalformation/). DWM becomes apparent in early infancy, is complicated by macrocephaly, and occurs along with increased intracranial pressure, spastic paraparesis, and hypotonia ${ }^{2}$. In addition, motor deficits, such as delayed motor development, hypotonia, and ataxia, as well as intellectual disability (ID), are often $\operatorname{seen}^{1,2}$. To date, various chromosomal abnormalities, such as trisomy 9 , $-13,-18$ and partial duplications/deletions of chromosomes, in DWM patients have been reviewed ${ }^{1}$. Additionally, heterozygous deletions of cerebellum-specific Zinc-finger genes, ZIC1 and ZIC4, on chromosome 3q24 are associated with $\mathrm{DWM}^{3}$. X-linked DWM with ID is also caused by an AP1S2 mutation ${ }^{4}$.

\footnotetext{
Correspondence: Yu-ichi Goto (goto@ncnp.go.jp)

${ }^{1}$ Department of Clinical Genome Analysis, Medical Genome Center, National Center of Neurology and Psychiatry (NCNP), Kodaira, Tokyo 187-8551, Japan ${ }^{2}$ Department of Child Neurology, National Center Hospital, NCNP, Kodaira, Tokyo 187-8551, Japan

Full list of author information is available at the end of the article.
}

OPHN1 encodes oligophrenin 1, which is a Rho-GTPase activating protein involved in synaptic morphogenesis and functions through the regulation of the $G$ protein cycle ${ }^{5}$. OPHN1 (NM_002547) consists of 25 exons and spans $\sim 391 \mathrm{~kb}$ on chromosome Xq12 (UCSC Genome Browser: https://genome.ucsc.edu). Oligophrein 1 is an 802 aminoacid protein harboring multiple domains, such as a BAR domain, PH domain, Rho-GAP domain, and three proline-rich sequences ${ }^{6} . O P H N 1$ was originally identified as a disrupted gene by a translocation $t(X ; 12)$ in a female patient with mild mental retardation ${ }^{7}$. To date, 10 point mutations, four splicing mutations, six small insertion/ deletion or duplication mutations, and 17 chromosomal rearrangements in OPHN1 have been identified in patients with neurodevelopmental disorders, including cerebellar hypoplasia, intellectual disability (ID), epilepsy, seizure, ataxia and schizophrenia (Human Gene Mutation Database, Professional 2018.2). In addition, Schwartz et al. $^{6}$ recently expanded the clinical spectrum of OPHN1associated phenotypes in comparison to the phenotypes described in previous reports. Moortgat et al. ${ }^{8}$ also described four families with intellectual disability without cerebellar hypoplasia.

Here, we report a Japanese DWM boy carrying an intragenic deletion in OPHN1. 
The biobank at the National Center of Neurology and Psychiatry (NCNP) is a unique biorepository for neuropsychiatric, muscular, and developmental diseases in Japan (https://ncbiobank.org/index-e.html). We collected DNA samples, clinical information, and cell lines, with informed consent, from 583 families with neurodevelopmental diseases that were diagnosed between 2004 and 2016. The study was approved by the ethical committee of NCNP. The cell lines were developed by the immortalization of peripheral lymphocytes with Epstein-Barr virus.

We conducted the candidate gene approach on chromosome $\mathrm{X}$ to identify the causative gene in this patient. The following 19 known causative genes for XLID were analyzed by repeat expansion analyses (FMR1 and $F M R 2)$ and Sanger sequencing (PQBP1, ARX, MECP2, ATRX, RPS6KA3, IL1RAPL1, TM4SF2, PAK3, FACL4, OPHN1, AGTR2, ARHGEF6, GDI1, SLC6A8, FTSJ1, ZNF41, and $D L G 3)$. We performed direct sequencing of PCR amplicons using an ABI3730 capillary sequencer (Thermo Fisher Scientific, Waltham, MA, USA) according to the standard protocol. We determined the breakpoint by comparing the sequence of the patient with sequences from an unaffected person.

The patient (III-1) was a 2-year-old boy, and he was referred for developmental delay at the age of 11 months. His maternal uncle was affected with hydrocephalus (Fig. 1a). The boy was born through normal delivery without asphyxia at 39 weeks of gestation. His weight and head circumference were $2756 \mathrm{~g}(-0.6 \mathrm{SD})$ and $32 \mathrm{~cm}$ $(-0.9 \mathrm{SD})$, respectively. He acquired head control at 8 months, but he could not sit alone or speak words. Brain computed tomography $(\mathrm{CT})$ and brain magnetic resonance imaging (MRI) suggested DWM (Fig. 1b). When he was 1 year old, he received shunting for his hydrocephalus. At the age of 1 year and 3 months, his height, weight, and head circumference were $81 \mathrm{~cm}(+1.1 \mathrm{SD})$, $10.7 \mathrm{~kg}(+0.8 \mathrm{SD})$, and $47 \mathrm{~cm}(0 \mathrm{SD})$, respectively, and his developmental quotient (DQ) was 57.

After initial screening for an L1CAM mutation by sequencing and a gross copy number variation in chromosome $\mathrm{X}$ by a BAC-based array-CGH ${ }^{9}$, which were both negative, we performed mutation screening of 19 known causative genes for XLID in the patient. Consequently, we found an intragenic deletion in OPHN1 involving exon 11-15, which include the PH and GAP domains (Fig. 2a). To more precisely determine the mechanism of the deletion, we performed deletion mapping by PCR-based sequence-tagged site content mapping, followed by direct sequencing of the junction fragment. We narrowed the breakpoint region to a 437-bp PCR product amplified by a set of PCR primers derived from intron 10 and intron 15 (Supplementary Fig. 1). Direct sequencing of the PCR product containing the recombination breakpoint revealed that the deletion only occurred within a common

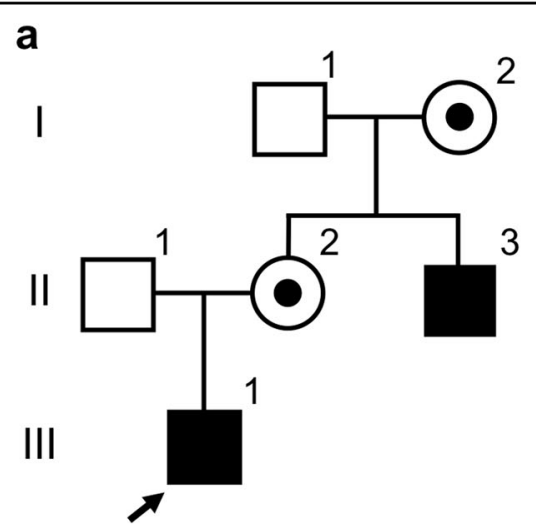

b

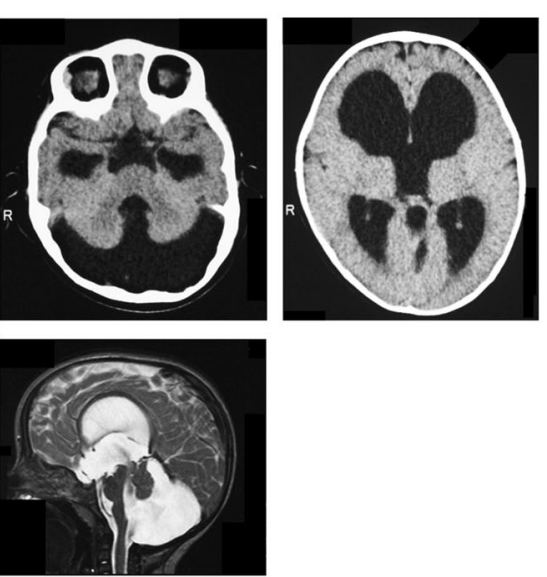

Fig. 1 The pedigree and the brain images. a Pedigree of the family. Arrow indicates the proband. $\mathbf{b}$ Brain computed tomography images (Upper) and a T2-weighted magnetic resonance image (Lower) of the patient. Prominent cerebellar hypoplasia, an enlarged fourth ventricle and posterior fossa, and ventriculomegaly were noted

five-nucleotide motif (AATTA) in intron 10 and intron 15 , both in the patient and his mother. No low copy repeats or segmental duplications were found adjacent to the deletion breakpoints, suggesting that the genomic rearrangement occurred by a microhomology-mediated mechanism and not by non-allelic homologous recombination (Fig. 2b). The deletion spanned from 4218 nucleotides downstream of the exon 10-donor site (c.933 $+4,218)$ to 4081 nucleotides downstream of exon 15 (c.1276 + 4,081). The size of the deletion was 13,517 bp in length (GRch37/hg19: chrX:67,408,680-67,422,196). This deletion was also absent in three public databases, dbVar, ClinVar and the Database of Genomic Variants (http:// dgv.tcag.ca/dgv/app/home). In addition, we did not find the deletion in a 1254 Japanese general population data set created by high-depth whole genome sequencing ${ }^{10}$.

In past studies, OPHN1 mutations have been reported in cases with XLID with cerebellar hypoplasia, strabismus, epilepsy, hypotonia, ventriculomegaly, and distinctive 


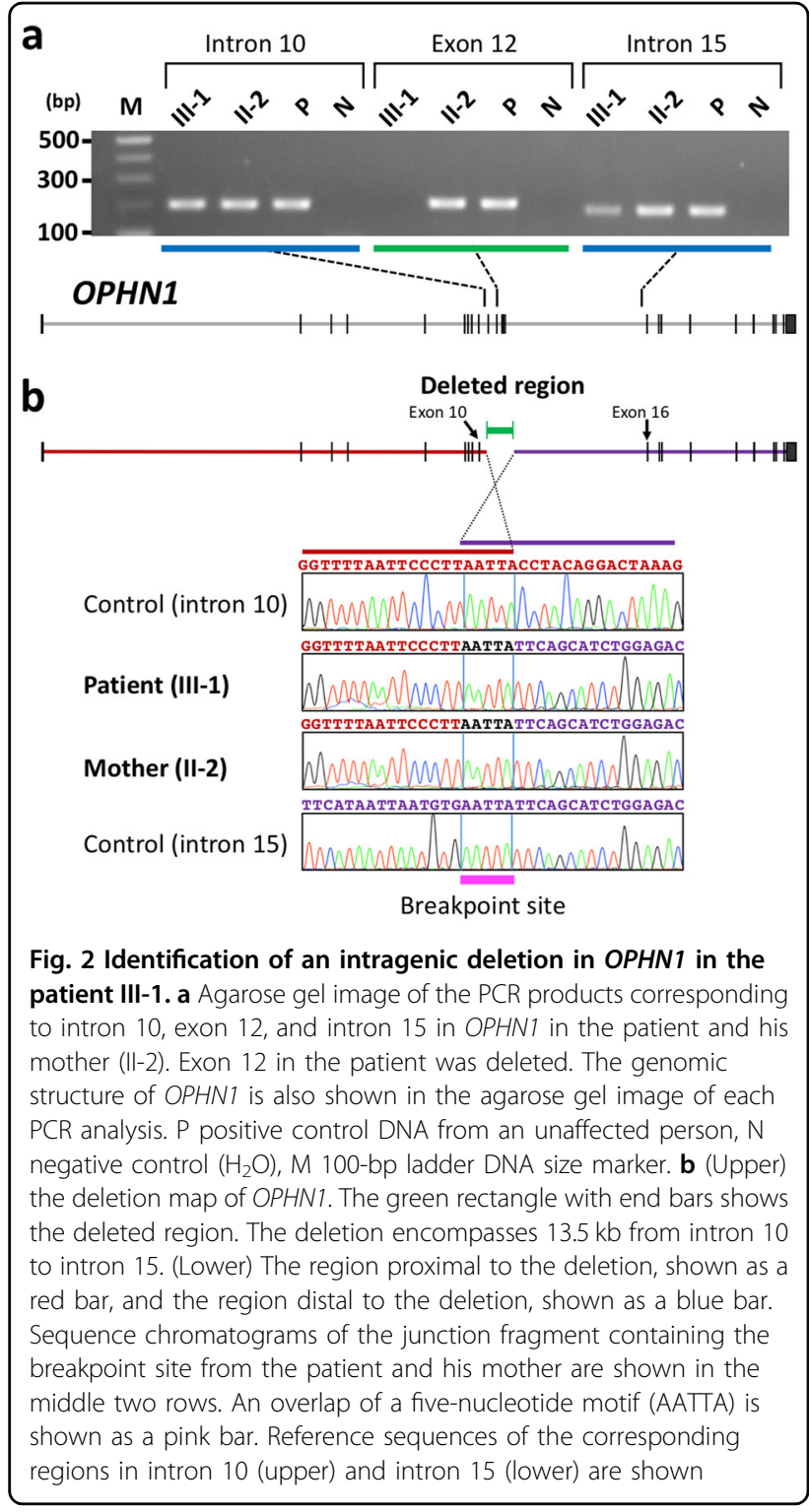

facial features ${ }^{6,8}$. Additionally, OPHN1 mutations have also been reported in individuals with autism or childhood onset schizophrenia $^{11}$, so OPHN1-associated clinical phenotypes are variable ${ }^{6-8,11}$. DWM and hydrocephalus in the present patient are likely the most severe imaging findings observed in patients with OPHN1 mutations. The novel intragenic deletion in OPHN1 eliminated exon 11-15, which encode $\mathrm{PH}$ and GAP domains. This deletion leads to a premature truncation, c.934_1276del (p.Gly312Ilefs*24), of OPHN1; the transcript might presumably be degraded by nonsensemediated mRNA decay. Altogether, we concluded that the deletion in OPHN1 is the pathogenic genetic abnormality in this patient who showed profound ID and DWM.

\section{HGV database}

The relevant data from this Data Report are hosted at the Human Genome Variation Database at

https://doi.org/10.6084/m9.figshare.hgv.2405

\section{Acknowledgements}

We are very grateful to the family who participated in this study. We thank Yoshie Sawano and Shoko Watanabe for their technical assistance. We also thank Dr. Johji Inazawa for the BAC-based array-CGH analysis. This study is partially supported by the Program for an Integrated Database of Clinical and Genomic Information (17kk0205012h0002 to Y. Goto), the Construction of integrated database of clinical and genomics information, and the sustainable system for promoting genomic medicine in Japan (18kk0205012s0303 to Y. Goto) from the Japan Agency for Medical Research and Development, AMED, and the Intramural Research Grant (27-6 to Y. Goto; 30-9 to A. lida) for Neurological and Psychiatric Disorders of NCNP.

\section{Author details}

${ }^{1}$ Department of Clinical Genome Analysis, Medical Genome Center, National Center of Neurology and Psychiatry (NCNP), Kodaira, Tokyo 187-8551, Japan. ${ }^{2}$ Department of Child Neurology, National Center Hospital, NCNP, Kodaira, Tokyo 187-8551, Japan. ${ }^{3}$ Laboratory for Statistical Analysis, RIKEN Center for Integrative Medical Sciences, Yokohama 230-0045, Japan. ${ }^{4}$ Kyoto-McGill International Collaborative School in Genomic Medicine, Kyoto University Graduate School of Medicine, Kyoto 606-8507, Japan. ${ }^{5}$ Laboratory for Genotyping development, RIKEN Center for Integrative Medical Sciences, Yokohama 230-0045, Japan. ${ }^{6}$ RIKEN Center for Integrative Medical Sciences, Yokohama 230-0045, Japan. ${ }^{7}$ Division of Medical Genetics, Kanagawa Children's Medical Center, Yokohama, Kanagawa 232-8555, Japan. ${ }^{8}$ Department of Mental Retardation and Birth Defect Research, National Institute of Neuroscience, NCNP, Kodaira, Tokyo 187-8551, Japan. ${ }^{9}$ Department of Bioresource, Medical Genome Center, NCNP, Kodaira, Tokyo 187-8551, Japan

\section{Conflict of interest}

The authors declare that they have no conflict of interest.

\section{Publisher's note}

Springer Nature remains neutral with regard to jurisdictional claims in published maps and institutional affiliations.

Supplementary information is available for this paper at https://doi.org/ 10.1038/s41439-018-0032-8.

Received: 1 October 2018 Revised: 25 October 2018 Accepted: 2 November 2018.

Published online: 5 December 2018

\section{References}

1. Parisi, M. A. \& Dobyns, W. B. Human malformations of the midbrain and hindbrain: review and proposed classification scheme. Mol. Genet. Metab. 80, 36-53 (2003).

2. Stambolliu, E., loakeim-loannidou, M., Kontokostas, K., Dakoutrou, M. \& Kousoulis, A. A. The most common comorbidities in Dandy-Walker syndrome patients: a systematic review of case reports. J. Child Neurol. 32, 886-902 (2017).

3. Grinberg, I. et al. Heterozygous deletion of the linked genes ZIC1 and ZIC4 is involved in Dandy-Walker malformation. Nat. Genet. 36, 1053-1055 (2004).

4. Cacciagli, P. et al. AP1S2 is mutated in X-linked Dandy-Walker malformation with intellectual disability, basal ganglia disease and seizures (Pettigrew syndrome). Eur. J. Hum. Genet. 22, 363-368 (2014).

5. Nadif Kasri, N. \& Van Aelst, L. Rho-linked genes and neurological disorders. Pflugers Arch. 455, 787-797 (2008).

6. Schwartz, T. S. et al. Expanding the phenotypic spectrum associated with OPHN1 variants. Eur. J. Med. Genet. S1769-7212, 30005-3 (2018).

7. Billuart, P. et al. Oligophrenin-1 encodes a rhoGAP protein involved in X-linked mental retardation. Nature 392, 923-926 (1998). 
8. Moortgat, S. et al. Expanding the phenotypic spectrum associated with OPHN1 mutations: report of 17 individuals with intellectual disability but no cerebellar hypoplasia. Eur. J. Med. Genet. S1769-7212, 30666-3 (2018).

9. Hayashi, S. et al. Construction of a high-density and high-resolution human chromosome $X$ array for comparative genomic hybridization analysis. J. Hum. Genet. 52, 397-405 (2007).
10. Okada, Y. et al. Deep whole-genome sequencing reveals recent selection signatures linked to evolution and disease risk of Japanese. Nat. Commun. 9 1631 (2018)

11. Piton, A. et al. Systematic resequencing of X-chromosome synaptic genes in autism spectrum disorder and schizopherinia. Mol. Psychiatry 16, 867-880 (2011). 